\title{
A User Centered Design Approach for Patient Interfaces to a Diabetes IT Platform
}

\author{
Giuseppe Fico ${ }^{1}$, Alessio Fioravanti ${ }^{1}$, Maria Teresa Arredondo ${ }^{1}$, Jan-Paul Leuteritz ${ }^{2}$, Alejandra Guillén, Duarte Fernandez ${ }^{3}$ \\ ${ }^{1}$ Life Supporting Technologies, Telecommunication Engineering School, Technical University of Madrid, Spain \\ ${ }^{2}$ Human Factors Engineering, Institute for Human Factors and Technology Management, University of Stuttgart, Germany \\ ${ }^{3}$ Medtronic Ibérica
}

\begin{abstract}
Improving patient self-management can have a greater impact than improving any clinical treatment (WHO). We propose here a systematic and comprehensive user centered design approach for delivering a technological platform for diabetes disease management. The system was developed under the METABO research project framework, involving patients from 3 different clinical centers in Parma, Modena and Madrid.
\end{abstract}

\section{INTRODUCTION}

$\mathrm{I}_{\mathrm{n}}$ ncorporating information technology in chronic disease management with a positive impact is an issue that still requires research efforts and investments. Health Information Systems (HIS) usually provide support related to guidelines, patient condition, and reminders to caregivers but this is not enough.

In 2007, Dorr et al. performed a systematic review of literature evaluating, from 1996-2005 (109 papers reviewed), informatics systems used in chronic disease management [1]: results of the literature analysis suggested that treatment adherence was positive, a decision support system was helpful in providing prompts but it was not helpful if it only focused on providing access to guidelines. Guidelines adherence was the most evaluated process with $79 \%$ of positive results. A large amount of studies came up with usability recommendations but a formal usability approach was adopted few times: we thus propose a systematic and comprehensive user centered design approach for delivering a technological platform for diabetes disease management.

\section{MATERIALS AND METHODS}

The aim of User Centered Design methodology is to satisfy the needs, the expectations and the satisfaction of the final users interacting with a system or a product, through their participation in the different phases of the development process. Three phases have been carried out:

1. Research Phase: user needs and main functionalities are extracted employing ethnographic techniques through interview and meeting with stakeholders, research on the state of the art and a market survey.

2. Concept Validation: a confrontation with users (patients, relatives, caregivers) is prepared to gather their insights on the concepts and extract their user demands and needs regarding interaction.

3. Development Phase: based on the previous point mockups are prepared and then validated through heuristic analysis by interaction experts.

Users are involved in all the phases through:

- Focus Group and Interviews: the goal is to gather information about the needs of patients related to the feedback strategy and contents.
- Usability Tests: to determine the usability of one prototype and to find ways to improve it ("formative testing"). DIN EN ISO 9241 and ISO 3407 has been used as the foundation of the test, as it is an internationally accepted standard. According to it, usability is "the extent to which a product can be used by specified users to achieve specified goals with effectiveness, efficiency and satisfaction in a specified context of use."

This is how usability can be measured, according to this standard:

- efficacy is the extent to which the user is able to achieve the respective goal(s). A common operationalisation of efficacy is the percentage of tasks completed successfully by the user.

- efficiency is the efficacy of the user, divided by the amount of resources the user needs to spend in order to reach this efficacy. A common measure of efficiency would therefore be the time to complete each task.

- satisfaction is the user's "subjective reaction" to the interaction with the product (ISO 9241). According to [4], user satisfaction is an emotion which results from the user comparing his expectations of the system to his actual experiences with it. Satisfaction can therefore only be measured by asking the user about his feelings towards the system.

The main results achieved for each of the phases are described as follows.

\section{STEP 1:THE PROBLEM}

\section{RESEARCH PHASE}

One of the first actions that were undertaken from the very beginning was to understand objectives and scope of the desired system: $70-90 \%$ of diabetics have insufficient glycemic control (HbA1c > 6.5-7\%). Major cause for inadequate control is a mismatch between lifestyle and treatment, but $<10 \%$ of type 1 diabetics are trained to adapt insulin doses to food/exercise and self-dosing training is not available for type 2 diabetics.

In terms of costs, the International Diabetes Federation (IDF) estimates that the equivalent of an additional 23 million years of life are lost each year to the disability and to reduced quality of life caused by the preventable complications of diabetes; in Europe IDF estimates [11] that the yearly cost of diabetes is around 67 Million International Dollars.

Clinicians can usually dedicate few minutes to each visit. It is currently difficult to predict which patient will be adherent. Many treatments fail because clinicians do not recognize noncompliant patients or a need of therapy scheme change. Meanwhile, patients frequently disagree with their 
physicians' plans and this is a major cause of a lack of compliance [2] since patients have veto power over doctor recommendations. $50 \%$ of patients leave office without having understood what their doctors told them to do [5]. About $50 \%$ of diabetic patients are not compliant with medications [6].

Care of diabetes is mainly based on drug administration at dosages which depend on the glucose level measured at fixed times during the day. These discrete measurements however do not allow calibrating correctly the drug dosage and even the most recent technologies for automatic insulin level measurement and drug infusion do not allow an optimal therapy assessment, being semi-invasive devices which patients wear only when required.

A team composed by industrial leaders in the field of $\mathrm{HC}$ technologies (Medtronic, others) defined, together with clinicians (University of Parma, Local Health Unit of Modena, Hospital San Carlos of Madrid, Charles University in Prague) and IT researchers (human computer interaction experts, biomedical engineers, authors of this paper), the open-ended approach with the aim of providing an in-depth study about the users in their natural environment. The result of this first step was the definition of SIX SEGMENTS (1. Changes in the environment, 2. Physical exercise, 3. Sudden hypoglycemic events, 4. Lack of motivation, 5. Unstable diabetes control, 6. Co-morbidity disease management): these are areas of focus representing what, according to ethnographic techniques, is defined as "the user natural environment within context of a specific process, situation, occasion, product and service. It's more than just another qualitative study" [7]: this categorization does not center in patients phenotypization, but addresses some specific situations in which patients may develop specific needs. It was under this framework that the further research was carried out:

\section{STEP 2: USER NEEDS}

Insights were gathered through meetings with clinicians, market analysis and technology experts, reflecting the study of the different user requirements to address each segment in order to set the technological feasibility to develop a system that can contribute to a better control of the diabetes disease management.

Not only a review of the particular user needs related to each segment was done, but also a general description of the basic features that the system could have provided for the six segments and that are common for all the users, independently of their profile. They can be summarized as:

- Acquisition of information from glucose readings and to the patient's lifestyle: food intake, drug intake, physical activity, etc.

- Acquisition of information related to complications and co-morbidities interacting with the diabetic disease

- Measurement of different variables related with the patient's ongoing treatment evolution such as weight, fat $\%$, etc.

- Design of a decision support system to assist in the recommendations for patients, enhancing empowerment and self-assurance of diabetic patients.

\section{STEP 3: FOCUSED RESEARCH}

Following the directives of the user needs, a State of the Art analysis was also focused in the six different segments, doing a research on the available technology that can be used by the system, identifying sensors and methodologies for the data acquisition.

Research was also carried out on data collection tools (for Indoor/Outdoor Scenarios, Fixed and Mobile, etc.). An investigation of the market needs was done in terms of innovation, support to patients and with an eye on the health strategies adopted by EU Member States' National Health Systems. The main determinants influencing success of telemedicine project implementation identified were:

Table 1 Factors influencing success in telemedicine projects

\begin{tabular}{|c|c|c|}
\hline Technology & Acceptance & Legislation \\
\hline $\begin{array}{l}\text { Support to users, both } \\
\text { in implementation and } \\
\text { operational phase in } \\
\text { installation, } \\
\text { maintenance and error } \\
\text { management; } \\
\text { Training in particular } \\
\text { if the system has an } \\
\text { high degree of } \\
\text { innovation; } \\
\text { Usability tailored on } \\
\text { skills of specific users, } \\
\text { mainly referring to } \\
\text { wearable or mobile } \\
\text { devices or access to } \\
\text { services; } \\
\text { Quality and reliability } \\
\text { of materials and } \\
\text { connections. }\end{array}$ & 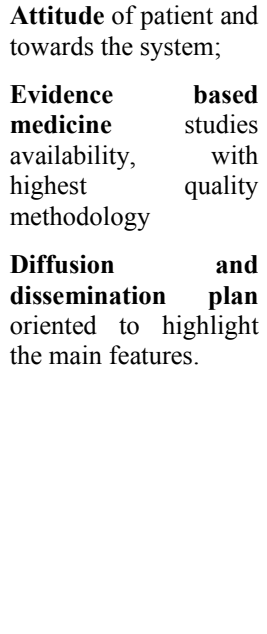 & $\begin{array}{l}\text { Current legislation } \\
\text { does not cover all the } \\
\text { aspect of telemedicine. } \\
\text { Legal standardization } \\
\text { is very difficult to } \\
\text { achieve at an } \\
\text { international level. } \\
\text { Member states legal } \\
\text { framework is not } \\
\text { homogeneous: major } \\
\text { barrier to wide-scale } \\
\text { system implementations } \\
\text { and increased device } \\
\text { "time to market". }\end{array}$ \\
\hline
\end{tabular}

The final steps that led to the Concept definition were:

- Meetings with physical activity in diabetes experts, diabetes psychologists and

- Interviews with patients

Users' applications were defined in terms of:

- Objectives

- What is expected from the application

- What is required from users

Every module of the application was defined from both clinical and technical points of view.

The main modules identified were:

- Glycaemic Module

- Food Intake

- Physical Activity

- Events Registration Module

- Diary

- Graphic Tools

- Motivation and Feedback Tool

STEP 4: CONCEPT VALIDATION

A confrontation with patients was prepared to gather their insights on the concepts and extract their user demands and needs regarding interaction. For this, interviews with 20 diabetic patients (16 T1DM, 4 T2DM) were prepared and completed. Interview methodology and strategy adopted has 
taken [8] and [9] as major references. The results obtained in these interviews were interpreted by a group of interaction designers to continue the process of refinement of all the concepts. General impressions gathered were:

- The concepts were generally very well accepted

- Special interest on activity reports and simulation tool before physical activity

- Major concern about the interaction time with the system

- Trust issues: most patients need to try the system themselves to prove it is reliable

- Good acceptance of feedback and recommendations, alerts and reminders but only if they were on demand (if they could be switched on and off)

- Major concern on the amount of devices to be needed

- Some users would appreciate using their own computers too.

\section{DEVELOPMENT PHASE}

Based on the work carried out in the research phase, the concept preparation and the first confrontations with patients and validation of the concepts, the main target users of the application were defined. These descriptions are based on the characterizations of the primary and secondary PERSONAs: PERSONAs are a precise descriptive model of the user, of what he or she wants to accomplish and why he or she wants to accomplish their goals. A well developed PERSONA comprises information on goals, attitudes, work or activity flow, environment, capacities and level of competence and frustrations. The definition of PERSONAs led to the creation of scenarios, which resume a regular day in the life of the PERSONAs and allow identifying the real needs. Scenarios were first created without the existence of the system and later, introducing it: through this approach, it was possible to recognize more easily the direct benefits of the use of the system that could be brought to the life of users.

As for patients, two PERSONAs were defined:

1. Ana, T1DM young patient insulin treated; most frequent tasks are related to the insertion of medical and biographical data. The functionalities provided by the system resemble the actions that the patient is used to perform, like the paper diaries or notebooks where patients take note of their measurements and comments. "Ana patients" have a fast and easy access to the data insertion functionalities. Patients have access to the diary and graphics areas from the home screen, but graphics and diary will not be shown directly on the home screen. Data reviewing is also a voluntary action. Feedback from health professionals should also be present at the home screen of the application. As most of this feedback will take place in the form of messages, the user will be able to see how many new, unread messages there are in the message inbox, as well as a direct access to the agenda where the application will show the next appointment or activity scheduled. Feedback takes place in the form of messages that are related with the correct usage and insertion of the information in the system.

2. George, T2DM aged patient not insulin treated. The focus should not be on data insertion, but on education, communication and empowerment to adopt a healthier lifestyle. Patients with similar characteristics to George most elderly T2DM patients - will usually be not insulin dependent, will suffer a number of co-morbidities and will probably have a complex medication regime. A special effort should be made in providing educational and motivational content, aiming to empower patients to change their routines and to adopt a healthier lifestyle. The application should be placed on lifestyle management and on feedback from health professionals. Most of the interaction to be defined for the application for elderly patients will be mainly application-driven, often started by the application by prompting messages and reminders.

\section{RESULTS}

\section{FOCUS GROUPS}

Focus groups with 23 patients (11 T1DM and 12 T2DM) were done in Madrid and Parma.

General results and lessons learned: Ana:

- The feedback messages needed to be reviewed and validated with patients and clinicians

- Physically intense labor had to be incorporated

- The dynamics of the focus had to be reviewed within frequent functions.

- The diary should include suggestions and indicate critical messages.

- The feedback decision support was indeed necessary, in order to give help when it's needed, e.g., when travelling

- Not all messages were to be kept stored in the system but only selected ones and for a selected period of time only. George:

- Not all messages were to be kept stored in the system but only selected ones and for a selected period of time only.

- The once proposed core-engine is indeed necessary, in order to provide the correct number and kind of messages at the correct time

- Diary and Advisor should rather be available as dialogue functions, associated to certain topics: medication, food, exercise, etc .

- The messages in the database needed to be reviewed. Some messages were changed, others were entirely taken out (especially those that were considered rude and not utile).

Common insights:

- The division into "Ana"- and "George"-users is necessary.

- The basic structure of Ana's PMD caters well for the users' needs.

- Providing real-time, data-based recommendations is wanted by all user groups.

- The most important topics are food and exercise (both, T1DM and T2DM).

Unlike other patients / elderly users, there are not any thoughts to reject aid-functions among diabetes patients.

\section{$\underline{\text { USABILITY TESTS }}$}


36 T1DM patients from Madrid, Modena and Parma were tested. The usability problems were reported to the development teams and have been addressed by them in the meantime. All in all, 43 different usability problems were identified within the tests. There were only few highly severe errors but a significant number of errors of medium severity, which needed to be addressed in order to assure a sufficient acceptance of the final application. The users' satisfaction with the "Ana"-Application was substantially higher than a medium score, which is the level of quality that we expect from this software.

Comments and wishes of the participants were also recorded. As users are neither designers nor technology experts, following their comments does not always lead to an increased usability. However, such comments should be checked when developing a user interface. We included these comments in our discussions when focusing on the improvement of the Ana-Application. We mention them here in order to give a complete picture of what was discussed, without putting all the details to each issue.

Table 2 - comments and wishes, concerning the "Ana"-Application

In the diary, the patient prefers most recent event at the top.

In the diary, the events should be ordered by their time of happening, not by the time they were entered.

About the red marked days in the monthly, agenda, patients suggested that they would show: Hypo (past events), Hyper (past events), sickness (past events), Visit to a Physician (future events), Agenda item (e.g., date)

Patients wished to see the reason for Hypo/hyper events

Patient preferred a correct relation between entering manual gr and adding more food by arrow on the virtual plate

"We calculate not in gr (CHO) but in "raciones" (this makes input more difficult and confuses in the main menu)

Some patients did not know how to calculate CHO or did not even know what that is.

The main screen should show a value that patients know: CHO in Italy, Raciones in Spain, ...

The participant preferred to visualize in the main screen $\mathrm{CHO}$ than $\mathrm{Kcal}$

It's not possible to erase old agenda items

As Figure 1 shows, the users' satisfaction with the "Ana"Application (calculated with the AttrakDiff questionnaire [12]) was substantially higher than a medium score, which is the level of quality that we expect from this software.

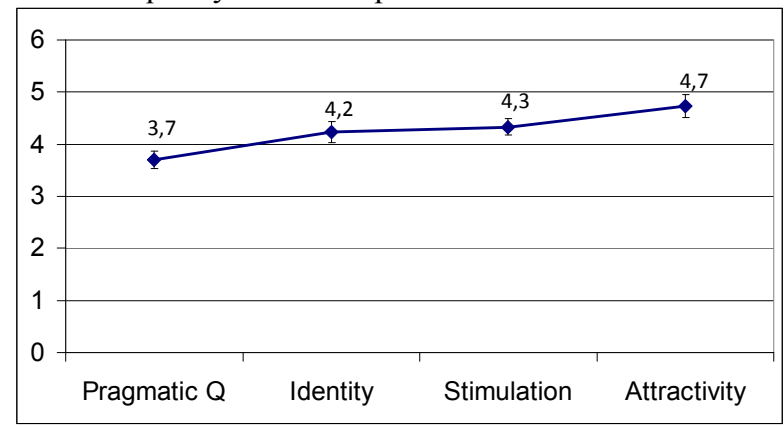

Figure 1 - Satisfaction with the "Ana"-Application

\section{CONCLUSIONS}

In this article we have described the process of defining patient interfaces for a complex telemedical platform system. We further depicted the outcomes of the different research steps indicating that diabetic patient vary in their specific needs regarding telemedical support, not only between patient subgroups but also intra-individually depending on the context and on the motivational status.

The METABO system is complex for two reasons: not only is Diabetes Mellitus a disease that require the control of a great variety of different lifestyle aspects; METABO also integrates many different data sources and actors. Therefore, the UCD process requires a broader methodology and recurrent involvement of end-users.

\section{ACKNOWLEDGMENT}

The authors wish to acknowledge the METABO consortium for their valuable contributions to this work. This project [10] is partially funded by the European Commission under the 7th Framework Program, Theme ICT-2007.5.1, Personal Health Systems for Monitoring and Point-of-Care Diagnostics - Personalized Monitoring, grant agreement number 216270

\section{REFERENCES}

[1] Informatics Systems to Promote Improved Care for Chronic Illness: a literature Review. Dorr et al, J Am Med Inorm Assoc. 2007;14:156-163. DOI 10.1197/jamia.M2255.

[2] Actively Engaging Patients in Treatment Decision Making and Monitoring as a Strategy to Improve Hypertension. Heisler, 2008 Americal Hearth Association, Inc. Circulation. 2008; 117:1355-1357.

[3] Hassenzahl, M., Burmester, M., Koller, F. (2003). AttrakDiff.: Ein Fragebogen zur Messung wahrgenommener hedonischer und pragmatischer Qualität. In Ziegler, J., \& Szwillus, G. (Eds.). Mensch \& Computer 2003 (pp. 187-196). Stuttgart: B. G. Teubner.

[4] Hassenzahl, M. (2004). Interaktive Produkte wahrnehmen, erleben, bewerten und gestalten. In Thissen, F. \& Stephan, P.F. (Eds.) Knowledge Media Design - Grundlagen und Perspektiven einer neuen Gestaltungsdisziplin. München: Oldenburg Verlag.

[5] Marvel et al. Soliciting the patient's agenda: have we improved? JAMA. 1999;281:283-287.

[6] Cramer. A systematic review o f adherence with medications for diabetes. Diabetes Care. 2004;27:1218-1224.

[7] "Ethnography and Innovation", Innovare ${ }^{\circledR}$ - (http://www.innovareinc.com/srv_ethno_techniques.htm)

[8] "A Practical Guide to Usability Testing - Paperback - J.S. Dumas, J. C. Redish - 1999

[9] "Measuring the User Experience" T. Tullis, B. Albert, Elsevier - 2008

[10] European Commission. Information Society Technologies Program. METABO project. Chronic diseases related to metabolic disorders. ICT26270. www.metabo-eu.org

[11] International Diabetes Federation (IDF), 2007. Diabetes Atlas. 3rd edition. Brussels: IDF. http://www.eatlas.idf.org/index1397.html, ISBN 2-930229-45-4, Hoorens Printing NV, Belgium

[12] Hassenzahl, M., Burmester, M., Koller, F. (2003). AttrakDiff.: Ein Fragebogen zur Messung wahrgenommener hedonischer und pragmatischer Qualität. In Ziegler , J., \& Szwillus, G. (Eds.). Mensch \& Computer 2003 (pp. 187-196). Stuttgart: B. G. Teubner 